Proceeding Series of the Brazilian Society of Computational and Applied Mathematics

\title{
Swing-By Propulsado aplicado ao sistema de Haumea
}

\section{Alessandra Ferraz da Silva Ferreira}

Departamento de Engenharia e Tecnologias Espaciais/ Mecânica Espacial e Controle, INPE, São José dos Campos, SP

\section{Antonio F. Bertachini de Almeida Prado ${ }^{2}$}

Departamento de Engenharia e Tecnologias Espaciais/ Mecânica Espacial e Controle, INPE, São José dos Campos, SP

\section{Othon Cabo Winter}

Grupo de Dinâmica Orbital e Planetologia, UNESP, Guaratinguetá, SP

\begin{abstract}
Resumo. O Swing-By propulsado é estudado. A manobra é aplicada ao sistema excêntrico de Haumea. O comportamento da energia do satélite é analisado. Os resultados mostraram condições onde a manobra é vantajosa em relação à aplicação do impulso e quanto pode ser obtido em termos de ganho de energia.
\end{abstract}

Palavras-chave. Swing-By, manobras impulsivas, trajetória de satélites, passagens próximas, impulso.

\section{Introdução}

Alguns trabalhos apresentados na literatura sobre manobra de Swing-By propulsada [2, 5-6] e sobre o problema elíptico restrito de três corpos [1].

O objetivo do presente trabalho é o estudo da manobra de Swing-By, para o caso onde os dois primários estão em órbitas elípticas, com a combinação da aplicação de um impulso em diferentes direções. É uma sequência do trabalho [3], que fez um estudo da manobra de Swing-By para o caso elíptico, porém sem a aplicação do impulso. Aplicações deste tipo de pesquisa podem ser generalizadas e aplicadas para qualquer sistema de primários excêntricos.

Um exemplo com alta excentricidade no Sistema Solar é o planeta anão Haumea. Considerando a presença de suas duas luas, podem-se formar os sistemas HaumeaHi'iaka e Haumea-Namaka. Eles foram estudados, pois se encaixam no problema

\footnotetext{
1 aleferrazsilva@hotmail.com

2 antonio.prado@inpe.br

3 ocwinter@gmail.com
} 
elíptico restrito de três corpos devido às altas excentricidades, principalmente de Namaka em relação à Haumea. Se um Swing-By propulsado é realizado em torno deles, a excentricidade da sua órbita deve ser levada em consideração e o seus efeitos são muito fortes. Haumea é um planeta anão localizado no Cinturão de Kuiper, a uma distância de 45 U.A. do Sol, com diâmetro equatorial de $1600 \mathrm{~km}$, tem rápida rotação e extrema elongação. Hi'iaka é sua maior lua, descoberta em janeiro de 2005, e a excentricidade de sua órbita é 0,0513. Sua massa estimada é de cerca de $1,79 \times 10^{19} \mathrm{~kg}$. Tem um diâmetro equatorial de aproximadamente $350 \mathrm{~km}$ e semi-eixo maior de $49880 \mathrm{~km}$. Namaka foi descoberta em junho de 2005, é interna a órbita de Haumea, tem excentricidade de 0,249 e massa estimada em $1,79 \times 10^{18} \mathrm{~kg}$, diâmetro equatorial de $170 \mathrm{~km}$ e semi-eixo maior de $25657 \mathrm{~km} \mathrm{[4].}$

Um algoritmo para o cálculo da variação da energia foi desenvolvido e aplicado a esses sistemas.

\section{Dinâmica do Sistema}

A dinâmica usada é a dada pelo problema restrito elíptico de três corpos, o que significa que o sistema é assumido ser formado por dois corpos massivos, chamado primários, em órbitas elípticas em torno do centro de massa deles e um terceiro corpo com massa negligenciável. É desenvolvido um algoritmo para obter a variação da energia para o Swing-By propulsado, que tem o impulso aplicado exatamente durante a máxima aproximação da passagem próxima. A direção do impulso pode ser variada. Desta maneira é possível encontrar a melhor direção para fazer a manobra impulsiva de modo a maximizar a variação da energia. Resultados de outros trabalhos mostram que a aplicação do impulso na direção do movimento do satélite geralmente não é a solução ótima e que há uma forte relação com a posição do corpo secundário na órbita em torno do corpo principal e com a excentricidade dos primários.

O sistema canônico de unidades é usado e abreviado como u.c. Os sistemas de referência mais usados são o fixo (inercial) e rotacional [7].

As equações do movimento do problema elíptico restrito de três corpos:

$$
\begin{aligned}
& \ddot{\bar{x}}=\frac{-(1-\mu)\left(\bar{x}-\bar{x}_{1}\right)}{r_{1}^{3}}-\frac{\mu\left(\bar{x}-\bar{x}_{2}\right)}{r_{2}^{3}} \\
& \ddot{\bar{y}}=\frac{-(1-\mu)\left(\bar{y}-\bar{y}_{1}\right)}{r_{1}^{3}}-\frac{\mu\left(\bar{y}-\bar{y}_{2}\right)}{r_{2}^{3}},
\end{aligned}
$$

Sendo $r_{1}$ a distância entre $M_{1}$ e $M_{3}$ e $r_{2}$ é a distância entre $M_{2}$ e $M_{3}$.

\section{Resultados}

O objetivo é estudar o efeito da aplicação do impulso exatamente no momento da 
máxima aproximação entre o veículo espacial e a lua de Haumea. Deseja-se obter qual a melhor direção para aplicação desse impulso, de modo a obter a máxima variação de energia possível.

\subsection{Haumea - Hi’iaka}

Para as simulações adotamos o valor de $\psi=90^{\circ}$ e $\psi=270^{\circ}$, anomalia verdadeira (v) de $\mathrm{M}_{2}$ no momento do encontro próximo igual a $0^{\circ}, 90^{\circ}, 180^{\circ}$ e $270^{\circ}$, magnitude do impulso de 0.5 a 2.0 unidades canônicas (u.c.), direção de aplicação do impulso $\alpha$ variando de $-180^{\circ}$ a $180^{\circ}$, com passo de 1,0 grau. A excentricidade de Hi'iaka em relação à Haumea é 0,0513.

O parâmetro de massa deste sistema é $\mu=0,004448$ e o raio do periapside foi adotado sendo 1,1 raios de Hi'iaka.

Figura 1 apresenta a variação da energia para os casos com $\psi=90^{\circ}$, impulso de 0.5 a 2.0 unidades canônicas e anomalia verdadeira $0^{\circ}$ (curva preta), $90^{\circ}$ (curva azul), $180^{\circ}$ (curva vermelha) e $270^{\circ}$ (curva verde). O eixo horizontal representa $\alpha$ (ângulo que define a direção do impulso) e o eixo horizontal representa a variação da energia.

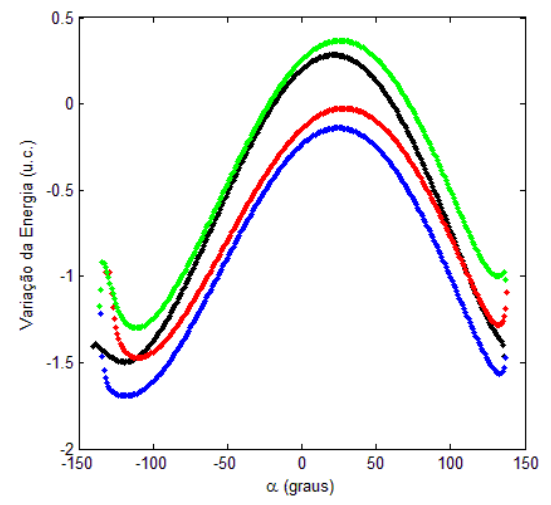

(a) $\delta \mathrm{V}=0.5$

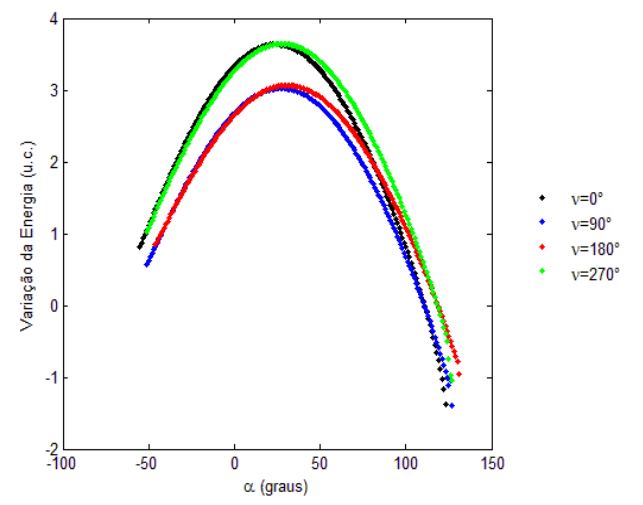

(c) $\delta \mathrm{V}=1.5$

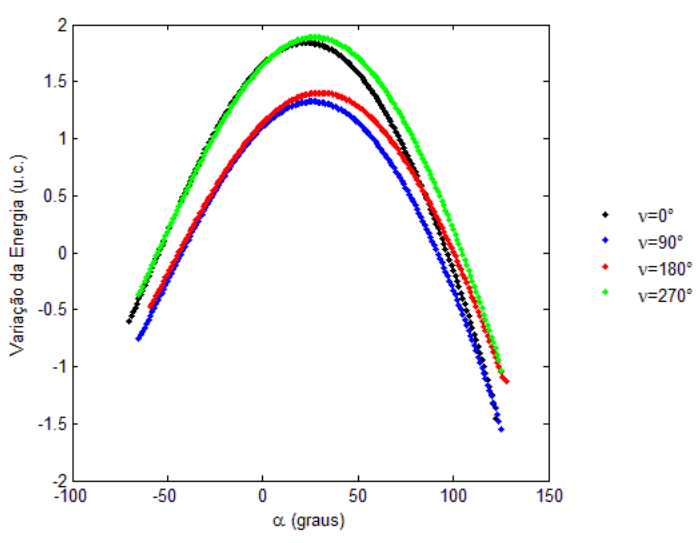

(b) $\delta \mathrm{V}=1.0$

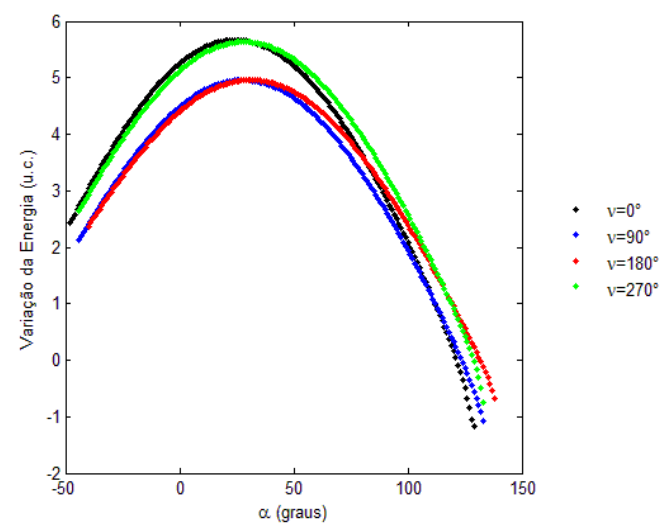

(d) $\delta \mathrm{V}=2.0$

Figura 1: Gráfico de $\alpha$ Vs variação da energia, para $\psi=90^{\circ}$, com diferentes $\delta \mathrm{V} \mathrm{e}$ valores da anomalia verdadeira $(v)$. 
A figura 2 apresenta a variação da energia para os casos $\operatorname{com} \psi=270^{\circ}$, impulso de 0.5 a 2.0 unidades canônicas e anomalia verdadeira $0^{\circ}$ (curva preta), $90^{\circ}$ (curva azul), $180^{\circ}$ (curva vermelha) e $270^{\circ}$ (curva verde). O eixo horizontal representa $\alpha$ (ângulo que define a direção do impulso) e o eixo horizontal representa a variação da energia.

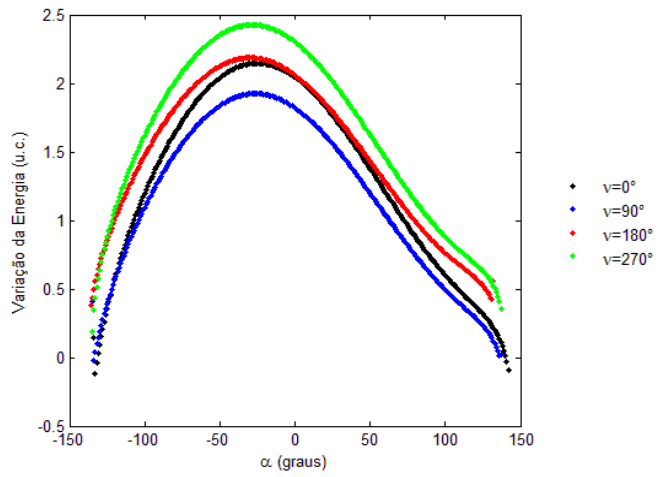

(a) $\delta \mathrm{V}=0.5$

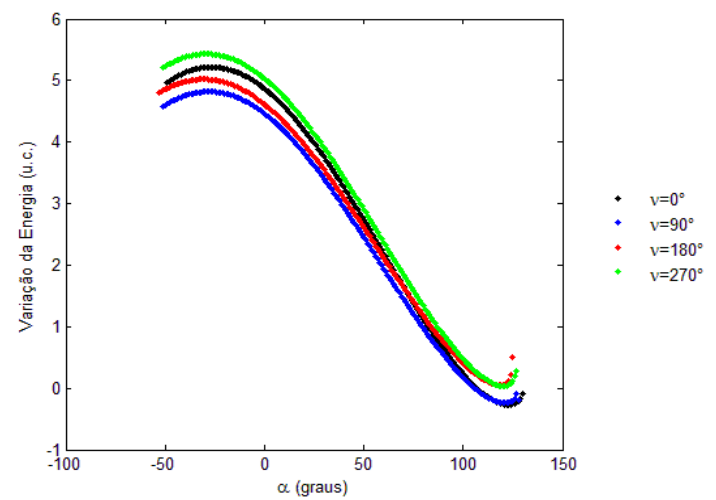

(c) $\delta \mathrm{V}=1.5$

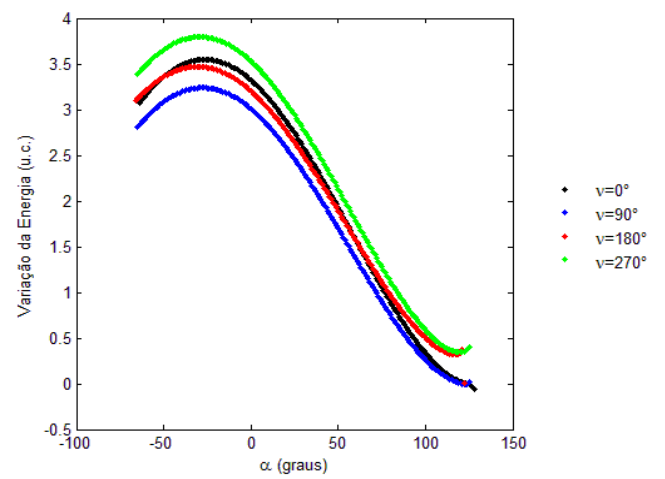

(b) $\delta \mathrm{V}=1.0$

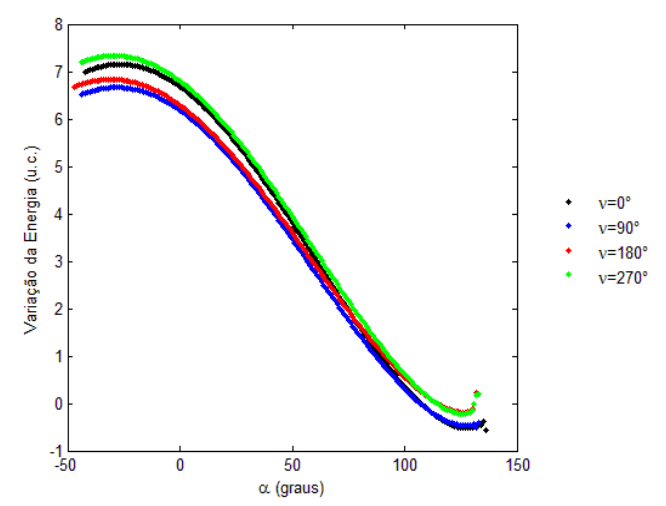

(d) $\delta \mathrm{V}=2.0$

Figura 2: Gráfico de $\alpha$ Vs variação da energia, para $\psi=270^{\circ}$, com diferentes $\delta \mathrm{V}$ e valores da anomalia verdadeira $(v)$.

Nota-se que o efeito da magnitude do impulso é o aumento na variação da energia. A excentricidade causa um aumento na velocidade orbital do satélite quando Hi'iaka está no periapside, e quando está no apoapside a velocidade é menor. Quando Hi'iaka está a $v=270^{\circ}$ de Haumea é a melhor posição para se obter maior variação na energia. O ganho máximo da energia ocorre em torno de $\alpha= \pm 20^{\circ}$. Na figura 2(d) a máxima variação de energia é 7.3303 u.c., $\alpha=-29^{\circ}$ e $v=270^{\circ}$. As regiões com descontinuidade em $\alpha$ são casos resultantes em capturas ou colisões.

\subsection{Haumea - Namaka}

O principal interesse neste estudo é o sistema Haumea-Namaka, devido à sua alta 
excentricidade, e $=0,249$. Adotamos o ângulo de aproximação $(\psi)$ igual a $90^{\circ}$ e $270^{\circ}$, anomalia verdadeira de $\mathrm{M} 2$ no momento do encontro próximo igual a $0^{\circ}, 90^{\circ}, 180^{\circ}$ e $270^{\circ}$, magnitude do impulso de 0.5 a 2.0 unidades canônicas (u.c.), $\alpha$ variando de $-180^{\circ}$ a $180^{\circ}$, com passo de 1,0 grau.

O parâmetro de massa deste sistema é $\mu=0,0004466$ e o raio do periapside foi adotado sendo 1,1 raios de Namaka.

A figura 3 apresenta a variação da energia para os casos $\psi=90^{\circ}$, com impulso de 0.5 a 2.0 unidades canônicas e anomalia verdadeira $0^{\circ}, 90^{\circ} 180^{\circ}$ e $270^{\circ}$, respectivamente. $\mathrm{O}$ eixo horizontal representa $\alpha$ (ângulo que define a direção do impulso) e o eixo horizontal a variação da energia.

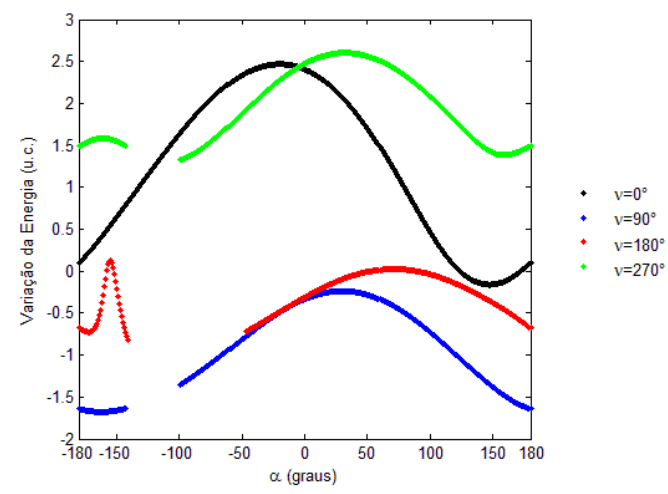

(a) $\delta \mathrm{V}=0.5$

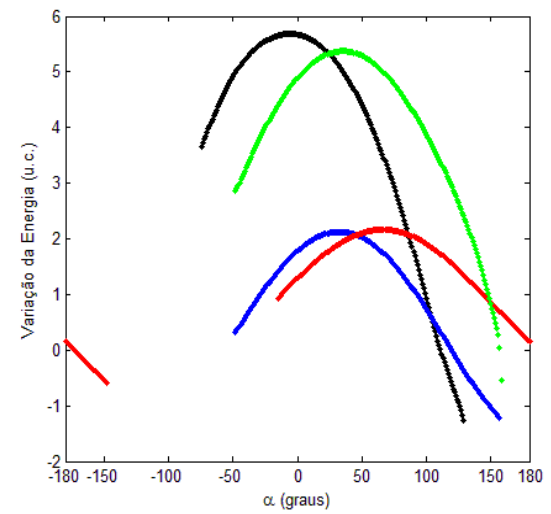

(c) $\delta \mathrm{V}=1.5$

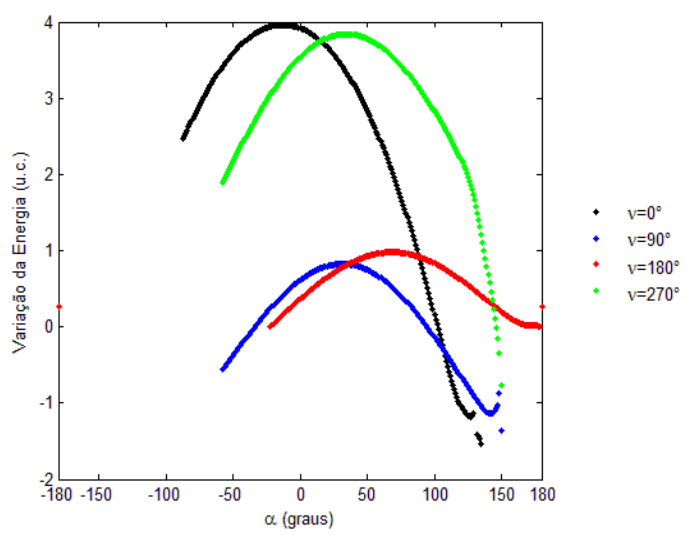

(b) $\delta \mathrm{V}=1.0$
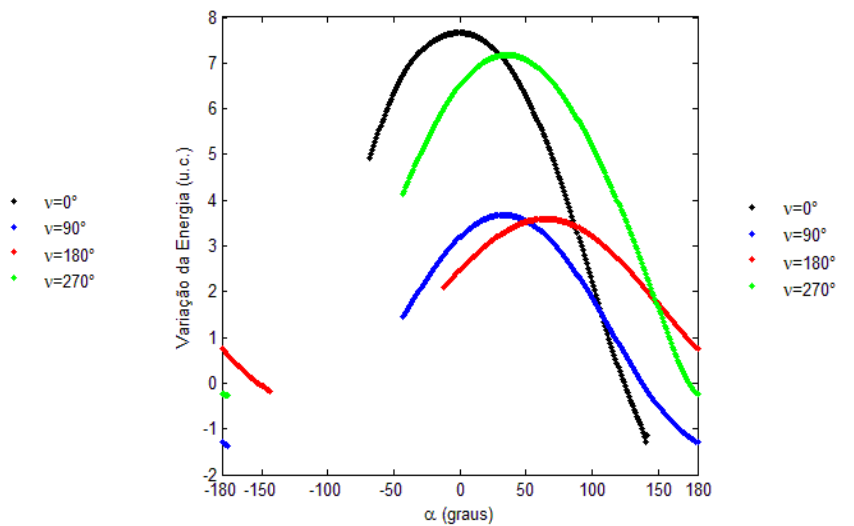

(d) $\delta \mathrm{V}=2.0$

Figura 3: Gráfico de $\alpha$ Vs variação da energia, para $\psi=90^{\circ}$, com diferentes $\delta \mathrm{V}$ e valores da anomalia verdadeira $(v)$.

A figura 4 apresenta a variação da energia para os casos com $\psi=270^{\circ}$, impulso de 0.5 a 2.0 unidades canônicas e anomalia verdadeira $0^{\circ}$ (curva preta), $90^{\circ}$ (curva azul), $180^{\circ}$ (curva vermelha) e $270^{\circ}$ (curva verde), respectivamente. O eixo horizontal representa $\alpha$ (ângulo que define a direção do impulso) e o eixo horizontal representa a variação da energia. 


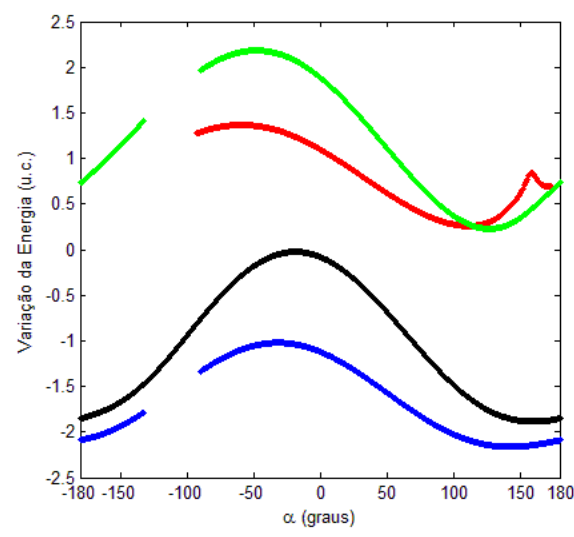

(a) $\delta \mathrm{V}=0.5$

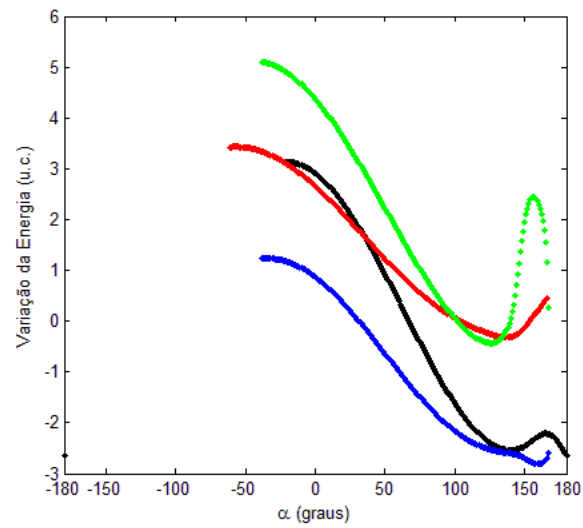

(c) $\delta \mathrm{V}=1.5$

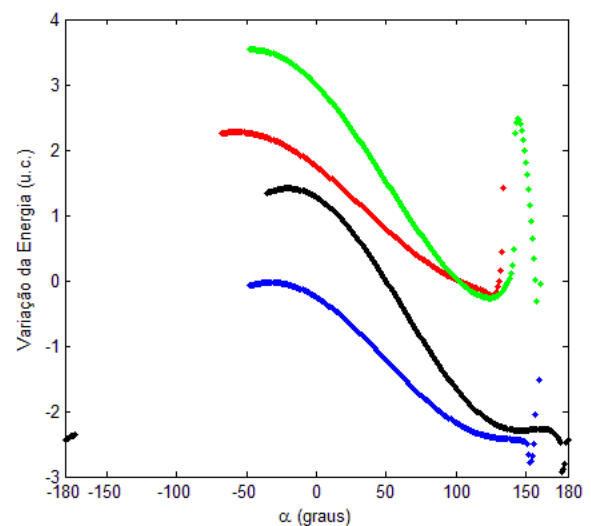

$\mathrm{v}=0^{\circ}$
$\mathrm{v}=90^{\circ}$

$\mathrm{v}=180^{\circ}$ $\mathrm{v}=270^{\circ}$

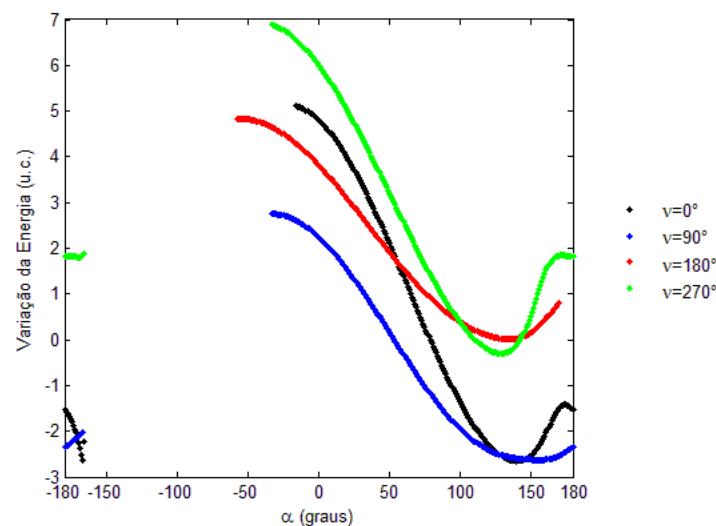

(d) $\delta \mathrm{V}=2.0$

Figura 4: Gráfico de $\alpha$ Vs variação da energia, para $\psi=270^{\circ}$, com diferentes $\delta \mathrm{V}$ e valores da anomalia verdadeira $(v)$.

A excentricidade neste caso é maior comparado ao caso anterior. Neste caso $v=90^{\circ}$ resulta em maior variação da energia. A magnitude do impulso é diretamente proporcional à variação da energia.

\section{Conclusão}

O trabalho mostra a variação da energia causada pela manobra de Swing-By propulsada aplicado ao sistema de Haumea, que é um planeta anão localizado no cinturão de Kuiper. O interesse nesse sistema é devido à excentricidade entre os primários. Foram analisadas quatro diferentes configurações entre os primários, sendo anomalia verdadeira igual a $0^{\circ}, 90^{\circ}, 180^{\circ}$ e $270^{\circ}$ e diferentes valores para a magnitude do impulso $(\delta \mathrm{V})$ e ângulo de aproximação $(\psi)$. Dos resultados vemos que a variação da energia é diretamente proporcional à magnitude do impulso. O efeito físico da excentricidade é que a velocidade orbital não é mais constante, a velocidade é maior quando $\mathrm{M}_{2}$ está no periapside e menor quando está no apoapside. No 
sistema Haumea-Hi' iaka a configuração que dá a maior variação de energia é quando $v=270^{\circ}$ e $\delta \mathrm{V}=2.0$ u.c. e para Haumea-Namaka é $v=90^{\circ}$ e $\delta \mathrm{V}=2.0$ u.c. Em geral, foi mostrado que a melhor direção para se aplicar o impulso nos casos estudados é na direção não tangencial a órbita $\left(\alpha \neq 0^{\circ}\right)$. Este estudo considerando a excentricidade entre os primários permite obter resultados mais realísticos do problema.

\section{Agradecimentos}

Os autores desejam expressar seu agradecimento pelo apoio prestado por doações \# 473387/2012-3, 304700/2009-6 e 312813/2013-9, do Conselho Nacional de Desenvolvimento Científico e Tecnológico (CNPq); subsídios \# 2011/13101-4, 2011/08171 3, 2012/21023-6 e 2014/06688-7 da Fundação de Pesquisa de São Paulo (FAPESP) e com o apoio financeiro do Conselho Nacional de Aperfeiçoamento de Ensino Superior (CAPES).

\section{Referências}

[1] R. A. Broucke, Stability of Periodic Orbits in the Elliptic, Restricted Three-Body Problem, AIAA Journal, vol. 7, 1003-1009, (1969).

[2] A. F. B.A. Prado, Powered swing-by, Journal of Guidance Control and Dynamics, vol. 19, 1142-1147, (1996).

[3] A. F. B. A, Prado, Close-approach trajectories in the elliptic restricted problem, Journal of Guidance, Control and Dynamics vol. 20, 797-802, (1997).

[4] D. Ragozzine, e M. E. Brown, Orbits and Masses of the Satellites of the Dwarf Planet Haumea (2003 EL61), Astronomical Journal, v. 137, 4766-4776, (2009).

[5] A. F. Silva, A. F. B. A. Prado, A.F.B.A. e O. C. Winter, Optimal impulsive control in a powered Swing-By, AIAA Guidance, Navigation, and Control (GNC) Conference, (2013).

[6] A. F. Silva, A. F. B. A. Prado, A.F.B.A. e O. C. Winter, Powered Swing-By Maneuvers around the Moon, Journal of Physics: Conference Series, vol. 465, (2013).

[7] V. Szebehely, Theory of Orbits, Academic Press, (1967). 